Nota técnica

Volumen 30(2):535-551. Mayo-agosto, 2019

e-ISSN 2215-3608, doi:10.15517/am.v30i2.33579

https://revistas.ucr.ac.cr/index.php/agromeso/index

\title{
Calibración y validación del equipo Veris MSP3 en dos suelos de Guanacaste, Costa Rica ${ }^{1}$
}

\section{Calibration and validation of Veris MSP3 on two soils in Guanacaste, Costa Rica}

\author{
Wanderson Novais ${ }^{2}$, José Carlos Rodríguez-Mejías², Johan Perret' ${ }^{2}$, Carlomagno Soto², José Eduardo Villalobos², \\ Carol Lucía Fuentes ${ }^{2}$, Karim Abdalla ${ }^{2}$
}

1 Recepción: 25 de junio, 2018. Aprobación: 8 de enero, 2019. Este trabajo formó parte del proyecto de graduación para obtener el título de Ingeniero Agrónomo con el grado académico de Licenciatura en Ciencias Agrícolas de los dos primeros autores, titulado: Aplicación y validación de técnicas de agricultura de precisión en arroz bajo condiciones de secano en Guanacaste, Costa Rica. Escuela de Agricultura de la Región Tropical Húmeda (EARTH), Limón, Costa Rica.

2 Escuela de Agricultura de la Región Tropical Húmeda (Universidad EARTH). Limón, Costa Rica. wnovais@earth.ac.cr, jcrodriguez@ earth.ac.cr (https://orcid.org/0000-0001-9843-9246),jperret@earth.ac.cr, csoto@earth.ac.cr, jvillalobos@earth.ac.cr, cfuentes@earth.ac.cr, kabdalla@earth.ac.cr

\section{Resumen}

Introducción. Es necesario implementar y validar tecnologías de agricultura de precisión (AP) en las regiones tropicales. Objetivo. El objetivo de este estudio fue calibrar y validar el equipo Veris MSP3 en dos terrenos distintos ubicados en la provincia de Guanacaste, Costa Rica. Materiales y métodos. Se correlacionaron los datos obtenidos por los sensores integrados en el equipo Veris MSP3 con análisis de laboratorio de muestras de suelo, y así desarrollar regresiones lineales simples o múltiples para predecir: textura del suelo, materia orgánica, nitrógeno en suelo, capacidad de intercambio catiónico total (CIC total) y pH, para obtener el modelo que mejor se adapte a cada parámetro. Resultados. Las regresiones que resultaron con los mejores modelos fueron: i) conductividad eléctrica aparente $(\mathrm{CEa})$ con textura del suelo, ii) radio óptico y pendiente con materia orgánica, iii) radio óptico con $\mathrm{N}$, iv) $\mathrm{CEa}$ con $\mathrm{CIC}$ total y v) $\mathrm{pH}$ Veris con $\mathrm{pH}$ en agua y $\mathrm{KCl}$. El coeficiente de determinación más alto se obtuvo entre CEa y porcentaje de arena con un $r^{2}$ de 0,82 . El $r^{2}$ para los parámetros evaluados varió de 0,28 a 0,82. Conclusión. El método de calibración utilizado obtuvo correlaciones razonablemente precisas $\left(\mathrm{r}^{2} \geq 0,55\right)$ para las variables textura de suelo en la profundidad de 0 a $30 \mathrm{~cm}$ y materia orgánica. Sin embargo, para textura de suelo de 30 a $90 \mathrm{~cm}, \mathrm{~N}$, CIC total y $\mathrm{pH}$, se debe considerar otra metodología para la calibración, debido a que se obtuvieron correlaciones no precisas $\left(r^{2} \leq 0,55\right)$.

Palabras clave: agricultura de precisión, conductividad eléctrica, $\mathrm{pH}$, reflectancia.

\begin{abstract}
Introduction. It is necessary to implement and validate precision agriculture (PA) technologies in tropical regions. Objective. The objective of this study was to calibrate and validate Veris MSP3 equipment in two different fields located in Guanacaste, Costa Rica. Materials and methods. The data obtained by the sensors integrated into the Veris MSP3 equipment was correlated with laboratory analysis of soil samples with the intent to develop simple or multiple linear
\end{abstract}


regressions to predict soil texture, organic matter, nitrogen in the soil, total cation exchange capacity, and $\mathrm{pH}$, in order to obtain a model that best suits each of the parameters. Results. The regressions that resulted with the best models were: i) apparent electrical conductivity (CEa) with soil texture, ii) optic ratio and slope with organic matter, iii) optic ratio with $\mathrm{N}$, iv) $\mathrm{CEa}$ with total cation exchange capacity (total CIC), and v) Veris $\mathrm{pH}$ with $\mathrm{pH}$ in water and $\mathrm{KCl}$. The higher determination coefficient was obtained between CEa and sand percentage with $r^{2}$ of 0,82 . In addition, the $r^{2}$ for the rest of parameters ranged from 0,28 to 0,82 . Conclusion. The calibration method used gave reasonably precise correlations $\left(\mathrm{r}^{2} \geq 0,55\right)$ for soil texture at depth from 0 to $30 \mathrm{~cm}$ and organic matter variables. However, from 30 to $90 \mathrm{~cm}$ soil texture, $\mathrm{N}$, total $\mathrm{CIC}$, and $\mathrm{pH}$ anoter calibration methodology should be considered because of imprecise correlations $\left(\mathrm{r}^{2} \leq 0,55\right)$.

Keywords: precision agriculture, electrical conductivity, $\mathrm{pH}$, reflectance.

\section{Introducción}

Una forma de medir la variabilidad espacial del suelo de manera indirecta es por medio de la conductividad eléctrica aparente (CEa), la cual puede correlacionarse con textura, humedad, salinidad, capacidad de intercambio catiónico, materia orgánica y otros. Las principales limitantes de la técnica son la necesidad de toma de muestras para establecer la correlación, así como previo conocimiento de los equipos que miden este parámetro en el suelo (Corwin, 2005). La CEa puede medirse de dos maneras, una por inducción electromagnética (EMI, en inglés) sin contacto con la superficie del suelo o por sensores de contacto que miden la resistencia (ER, en inglés), en donde discos emiten y reciben simultáneamente pulsos eléctricos (Corwin y Lesch, 2005; Grisso et al., 2009).

Entre los estudios realizados en CEa se pueden mencionar los de Williams y Hoey (1987), Weller et al. (2007) y Wetterlind et al. (2015) para componentes texturales en particular la arcilla, Yao et al. (2016) para salinidad y mapas de productividades, y Sudduth et al. (2005), Machado et al. (2015) y Wetterlind et al. (2015) con materia orgánica, capacidad de intercambio catiónico, humedad y conductividad eléctrica en pasta saturada. Los estudios mencionados fueron hechos con diferentes equipos, tales como: Geonics EM38MK2, Veris 3100 y GSSI EMP Profiler-400.

Con el avance de la agricultura de precisión (AP), se incrementó la necesidad de medir la variabilidad en el suelo de factores de producción como es el $\mathrm{pH}$ y la materia orgánica. No obstante, para $\mathrm{pH}$, varios estudios han demostrado que se puede encontrar variabilidad espacial en menos de $100 \mathrm{~m}$ de distancia (Mulla y McBrantney, 2000), inclusive, en algunos terrenos se ha determinado cambios de dos unidades de $\mathrm{pH}$ en menos de $12 \mathrm{~m}$ de distancia (Bianchini y Mallarino, 2002). Esto causa que el uso de técnicas tradicionales de muestreo requieran gran cantidad de muestras para delinear los cambios de pH (densidad alta de muestreo), volviéndose impráctico en términos de costos y tiempo. Basándose en la necesidad de detectar las diferencias en pH de manera precisa, Adamchuk et al. (1999) desarrollaron un sistema automatizado de medición de pH en movimiento.

En el caso de la materia orgánica, Maxton et al. (2017) desarrollaron un sensor espectrofotómetro de bajo costo con dos anchos de bandas, uno en infrarrojo (Vis-NIR, en inglés) y el otro en infrarrojo cercano (NIR, en inglés), para emisión de luz, además, de un fotodiodo que transformaba luz en corriente eléctrica, dando el resultado en reflectancia. Esta puede correlacionarse con materia orgánica analizada en laboratorio, utilizando una regresión lineal simple en el caso de suelos con alto contenido de materia orgánica (suelos negros), y regresión lineal múltiple para otros tipos de suelos.

Una carencia de investigaciones que sustente la aplicación comercial de AP en latitudes tropicales es indicada por Melo et al. (2014), a pesar de que ha incrementado la discusión sobre el tema. Entre los pocos trabajos disponibles, el de Hernández et al. (2006) mostraron una propuesta de fertilización dinámica en una empresa agro comercial de banano, basándose en mapeo de suelos, sustentaron un programa de fertilización cumpliendo los requerimientos nutricionales del cultivo y demostraron costos anuales menores a la fertilización convencional. 
Es necesario validar técnicas y equipos utilizados en agricultura de precisión. Este estudio tuvo como objetivo calibrar y validar el equipo Veris MSP3, en dos terrenos distintos ubicados en la provincia de Guanacaste, Costa Rica.

\section{Materiales y métodos}

La investigación para calibrar y validar el equipo Veris MSP3, con base en la medición de la variabilidad espacial del suelo de manera indirecta, por medio de la conductividad eléctrica aparente (CEa), se realizó en el año 2017 en dos terrenos agrícolas en la provincia de Guanacaste (Cuadro 1), Caimital de Nicoya, denominado como Costeña con un área de 8,89 ha y el segundo en Liberia en el campus La Flor de la Universidad EARTH, denominado CAP (Centro de Agricultura de Precisión) con 1,12 ha.

Cuadro 1. Descripción de los campos utilizados en el estudio de calibración y validación del equipo Veris MSP3, en dos suelos de Guanacaste, Costa Rica. 2017.

Table 1. Field descriptions in the study of calibration and validation of Veris MSP3 on two soils in Guanacaste, Costa Rica. 2017.

\begin{tabular}{|c|c|c|c|c|c|c|}
\hline Lote & Coordenadas $\ddagger$ & $\begin{array}{c}\text { Elevación } \\
(\mathrm{msnm}) \ddagger\end{array}$ & $\begin{array}{l}\text { Promedio anual de } \\
\text { temperatura }\left({ }^{\circ} \mathrm{C}\right) *\end{array}$ & $\begin{array}{c}\text { Promedio anual de } \\
\text { precipitación }(\mathrm{mm}) *\end{array}$ & $\begin{array}{c}\text { Orden del } \\
\text { suelo } t\end{array}$ & $\begin{array}{l}\text { Suborden } \\
\text { del suelot }\end{array}$ \\
\hline Costeña** & $10^{\circ} 05^{\prime} 12^{\prime \prime} \mathrm{N} 85^{\circ} 28^{\prime} .38^{\prime \prime} \mathrm{O}$ & 160 & 28,5 & 143,47 & Alfisol & Ustalfs \\
\hline $\mathrm{CAP} * * *$ & $10^{\circ} 38^{\prime} 55.92^{\prime \prime} \mathrm{N} 85^{\circ} 32^{\prime} 05.86^{\prime \prime} \mathrm{O}$ & 60 & 27,5 & 135,2 & Inceptisol & Ustepts \\
\hline
\end{tabular}

‡ Google EARTH (2017).

+ CIA (2016).

* IMN (2017).

**/ Caimital de Nicoya, provincia de Guanacaste / Caimital, Nicoya, Guanacaste province.

***/ Liberia en el campus La Flor, Centro de Agricultura de Precisión, Universidad EARTH / Campus La Flor, Liberia, Precision Agriculture Center, EARTH University.

Los suelos del estudio presentaron diferencias en términos de textura, en los primeros $30 \mathrm{~cm}$ de profundidad predominaron: arcilloso en Costeña y franco en CAP, para la profundidad de 30 a $90 \mathrm{~cm}$ fue arcilloso en Costeña $\mathrm{y}$ franco arcilloso para CAP.

El equipo Veris MSP3 está compuesto de tres partes fundamentales: el pH sampler, que mide el pH automatizado de contacto directo con el suelo, los discos para la medición de conductividad eléctrica aparente en $\mathrm{mS}^{-1} \mathrm{~m}^{-1}$ y por último, el sensor óptico que opera con dos anchos de banda vis-NIR y NIR (reflectancia) (Figuras 1 y 2).

Con las diferentes partes del equipo Veris MSP3 es posible medir la conductividad eléctrica aparente (CEa) en dos profundidades ( 0 a $30 \mathrm{~cm}$ y 30 a $90 \mathrm{~cm}$ ), reflectancia en dos anchos de banda, $\mathrm{pH}$ con el sensor antimonio y datos topográficos del terreno, además, todas sus mediciones se realizan mientras es remolcado por un tractor y georreferenciadas por un GPS interno.

En el terreno Costeña, el levantamiento de datos se realizó durante el mes de marzo (periodo seco en la región), con la humedad en el suelo que exige el equipo Veris MSP3 en las especificaciones (10\% por encima del punto de marchitez permanente). En el terreno del CAP, las lecturas se hicieron en el mes de setiembre (periodo lluvioso en Guanacaste).

El equipo Veris MSP3, realizó 381 lecturas por hectárea de CEa y reflectancias en Costeña, en 10 trayectos con un espaciamiento de $15 \mathrm{~m}$ entre líneas y $1,75 \mathrm{~m}$ entre lecturas en promedio. El equipo midió CEa en dos profundidades: superficial (shallow) de 0 a $30 \mathrm{~cm}$ (CEa-sh) y profunda (deep) de 0 a $90 \mathrm{~cm}$ (CEa-dp); para 


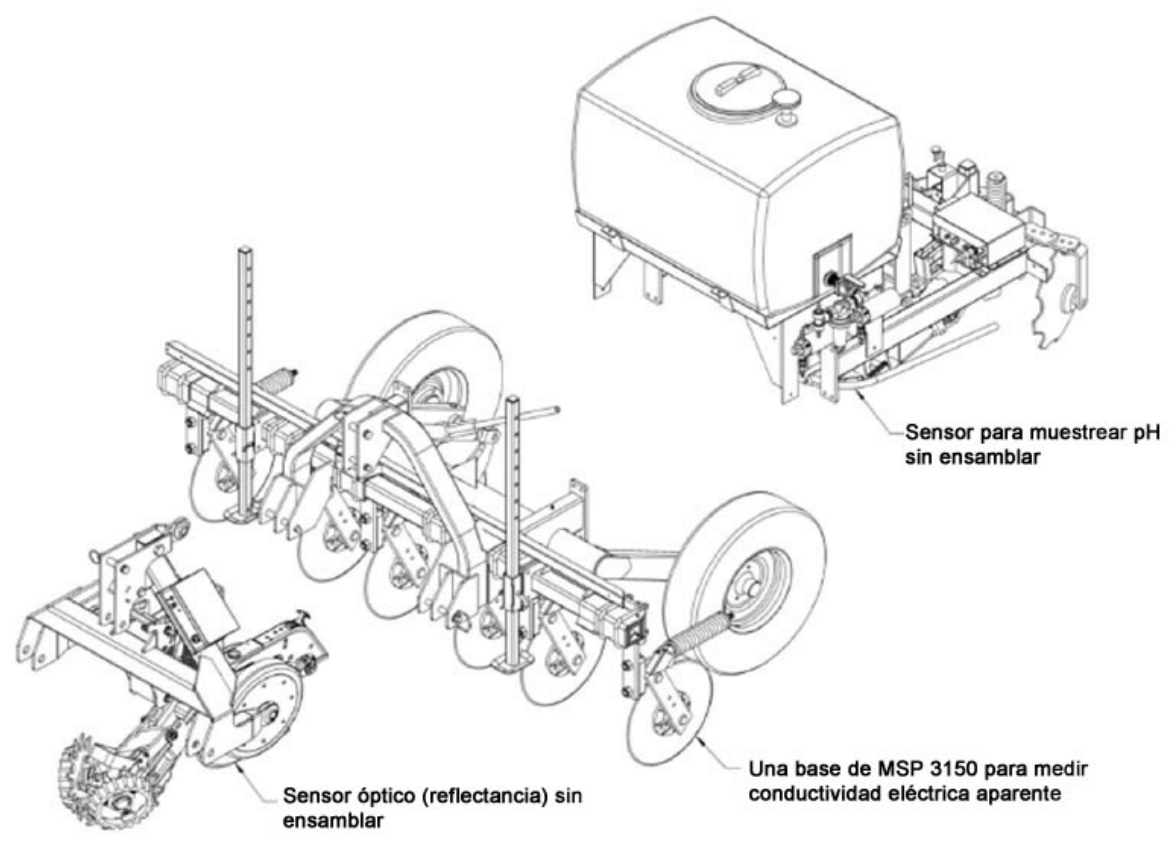

Figura 1. Partes del equipo Veris MSP3 para medir la conductividad eléctrica aparente (CEa), reflectancia en dos anchos de banda y pH. Adaptado de Veris Technologies (2017a).

Figure 1. Veris MSP3 parts to measure apparent electrical conductivity, reflectance in two bandwidths and pH. Adaptado de Veris Technologies (2017a).

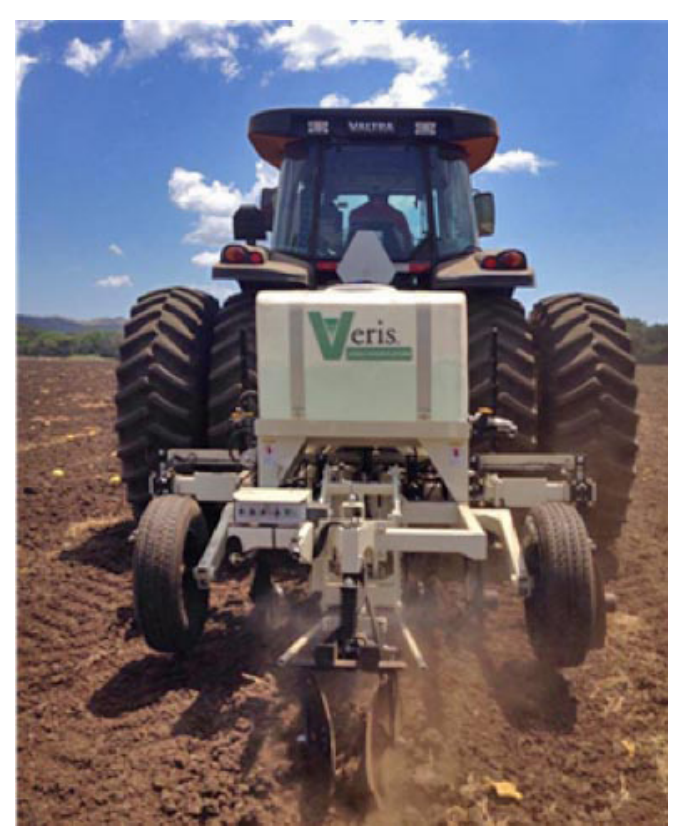

Figura 2. Equipo Veris MSP3 trabajando en el terreno Costeña, ubicado en Caimital de Nicoya, Guanacaste, Costa Rica. 2017.

Figure 2. Veris MSP3 equipment working in the Costeña field, located in Caimital, Nicoya, Guanacaste, Costa Rica. 2017. 
reflectancias las lecturas fueron a los 7,5 cm de profundidad. En CAP se obtuvieron 723 lecturas por hectárea de CEa y reflectancias, en 6 trayectos con espaciamiento de aproximadamente $15 \mathrm{~m}$ entre líneas y 1,5 m entre lecturas en promedio, en las mismas profundidades mencionadas.

Los datos recolectados en ambas localidades por el equipo se procesaron en el Veris Mapping Center (Centro de procesamiento de datos de la empresa Veris Technologies), para eliminar mediciones irregulares de campo, siendo esta la base de datos utilizadas para la calibración y validación de Veris, además de calcular e incorporar datos de curvatura y elevación.

Con el programa digital Surfer (2017), se elaboraron mapas interpolados con el método Kriging modelo lineal con 1200 por 1200 nodos de las variables CEa-sh (Figura 3), CEa-dp (Figura 4) y reflectancia de NIR en Costeña (Figura 5) y CAP (Figura 8). Para el muestreo de calibración en cada mapa interpolado, se seleccionaron los puntos de muestreo en zonas de contorno bien estructuradas (uniformes), evitando zonas de transición, esto con el fin de implementar un diseño de muestreo estratificado (Johnson et al., 2001) en ambos sitios de estudio (Figuras de la 3 a la 8) buscando abarcar de dos a tres muestras por estrato.

De las muestras colectadas de suelo, en el laboratorio se procedió a realizar un análisis de textura con el metodo Bouyoucos en las dos profundidades para correlacionar con CEa, mientras que para NIR y CEa-sh se analizaron porcentaje de materia orgánica (MO), nitrógeno y capacidad total de intercambio catiónico (CIC total).

El pH se midió solo en CAP en dos diferentes modos: semi controlado $(\mathrm{smc})$ y on the go, siguiendo la metodología de Schirrmann et al. (2011). El modo smc consistió en ir moviéndose con el equipo en un trayecto, realizar paradas en el terreno para obtener una lectura con el sensor y, al mismo tiempo, tomar la misma muestra de suelo para el análisis en laboratorio. El modo on the go consistió en el manejo normal del Veris (avanzando en trayectos y tomando muestras al mismo tiempo).

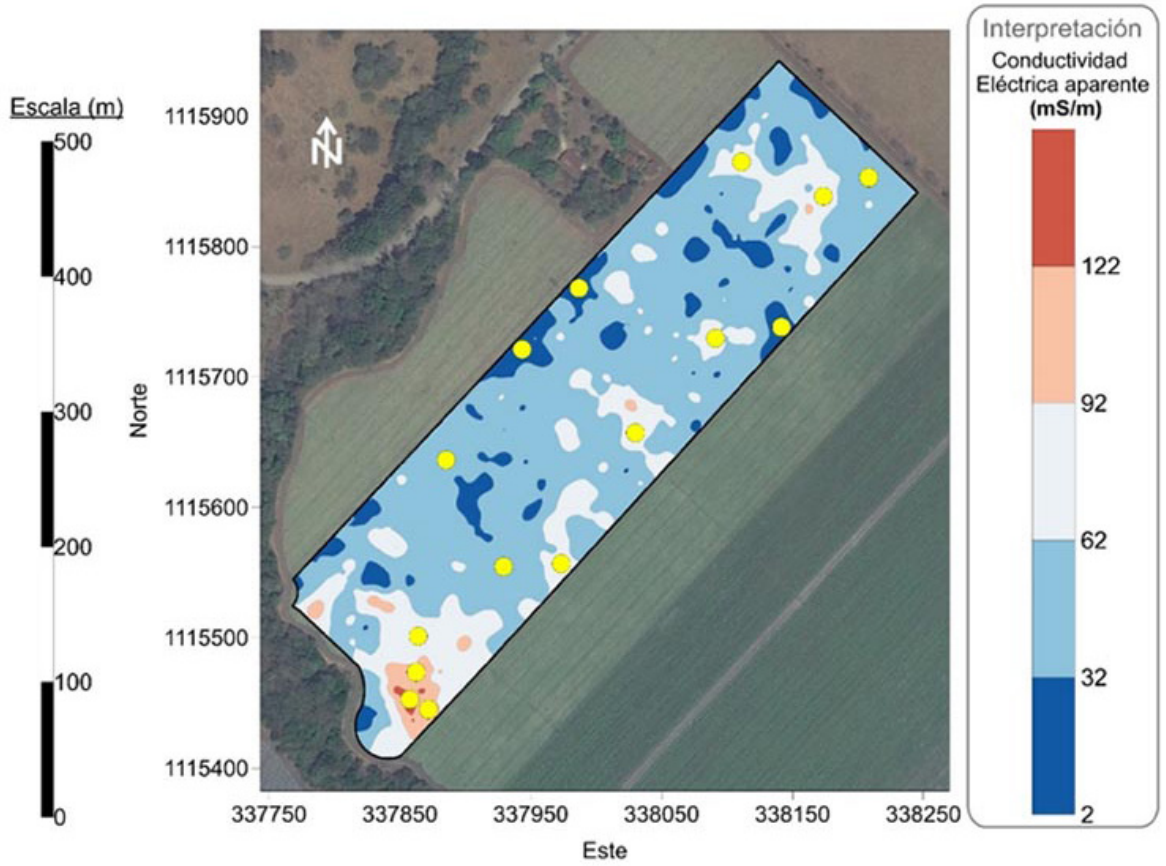

Figura 3. Mapa de conductividad eléctrica aparente superficial (CEa-sh, 0 a $30 \mathrm{~cm}$ de profundidad) y puntos de muestreo en amarillo para calibración en Costeña, ubicada en Caimital de Nicoya, Guanacaste, Costa Rica. 2017.

Figure 3. Shallow apparent electrical conductivity map (ECa-sh, depth of 0 to $30 \mathrm{~cm}$ ) and samples sites for calibration in yellow in Costeña, located in Caimital, Nicoya, Guanacaste, Costa Rica. 2017. 


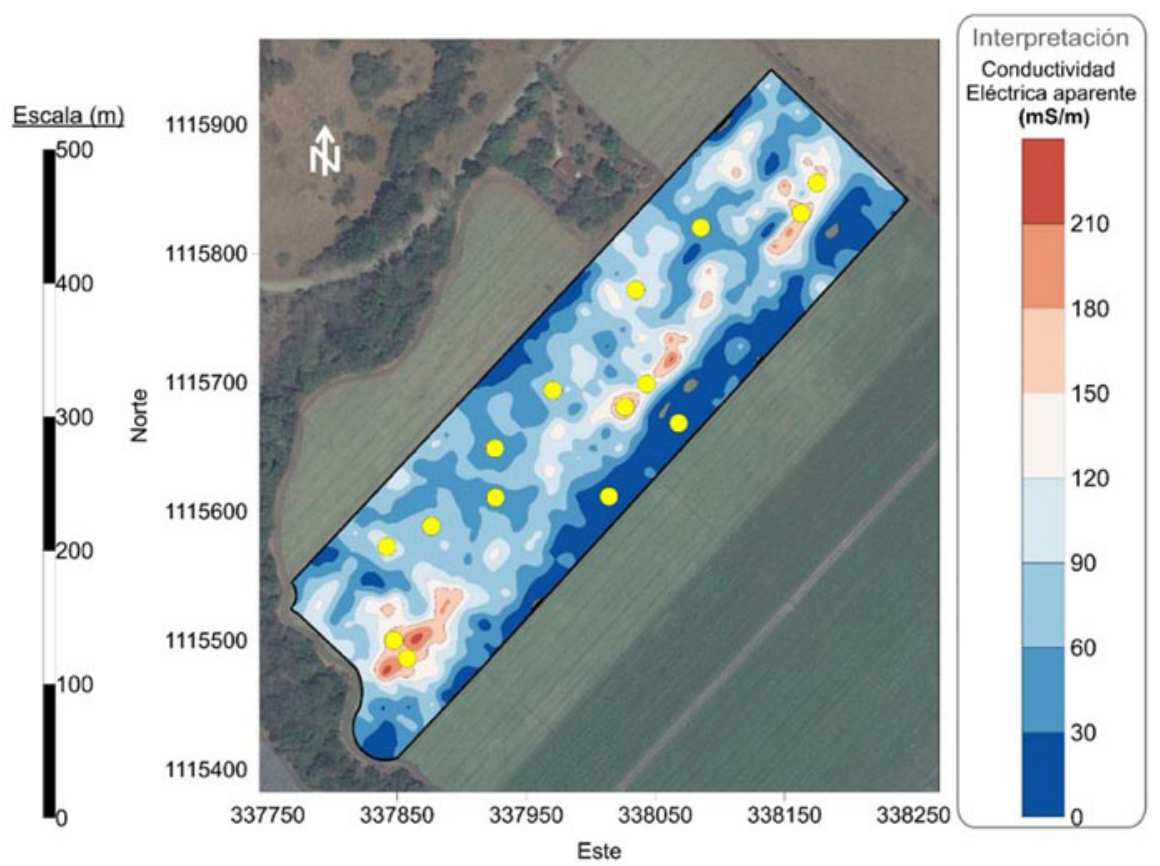

Figura 4. Mapa de conductividad eléctrica aparente profunda (CEa-dp, de 30 a $90 \mathrm{~cm}$ de profundidad) de Costeña y puntos de muestreo en amarillo para calibración, ubicada en Caimital de Nicoya, Guanacaste, Costa Rica. 2017.

Figure 4. Deep apparent electrical conductivity map (ECa-dp, depth of 30 to $90 \mathrm{~cm}$ ) of Costeña and samples sites for calibration in yellow, located in Caimital, Nicoya, Guanacaste, Costa Rica. 2017.

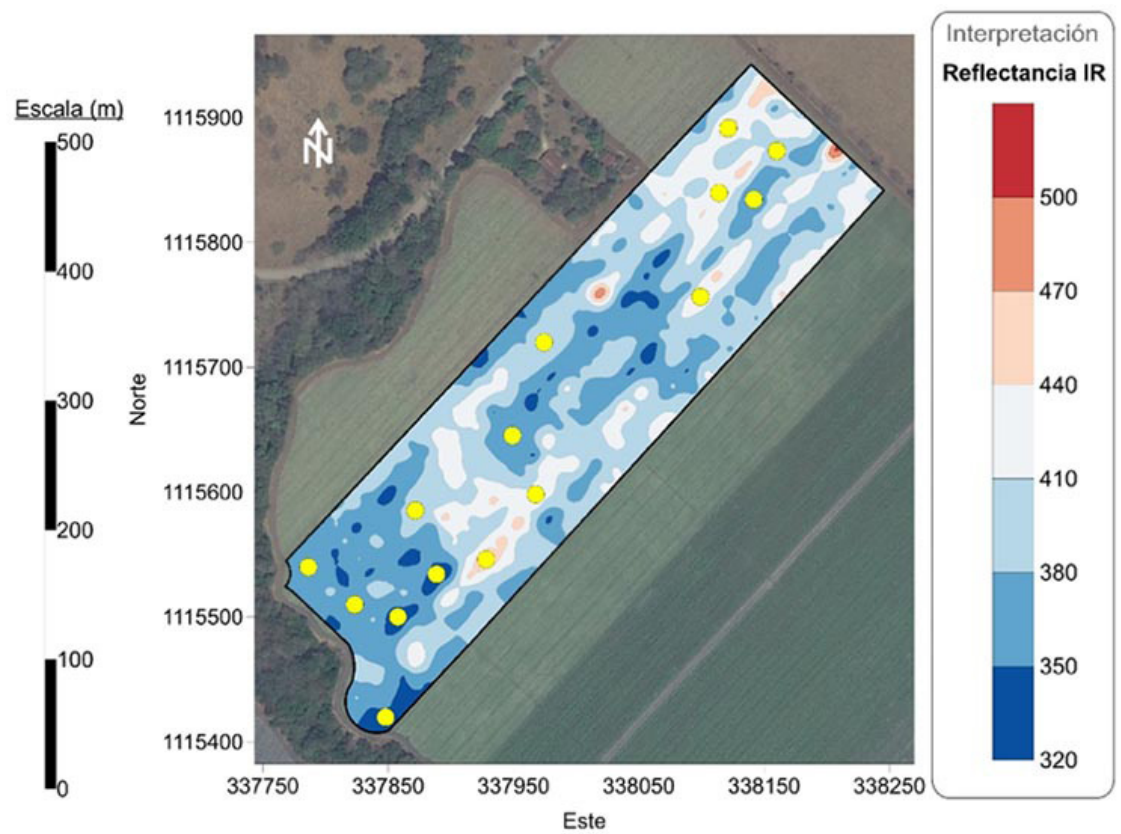

Figura 5. Mapa de reflectancia en infrarrojo cercano (NIR) en Costeña y puntos de muestreo en amarillo para calibración, ubicada en Caimital de Nicoya, Guanacaste, Costa Rica. 2017.

Figure 5. Near-infrared reflectance (NIR) map of Costeña and calibration samples sites in yellow, located in Caimital, Nicoya, Guanacaste, Costa Rica. 2017. 


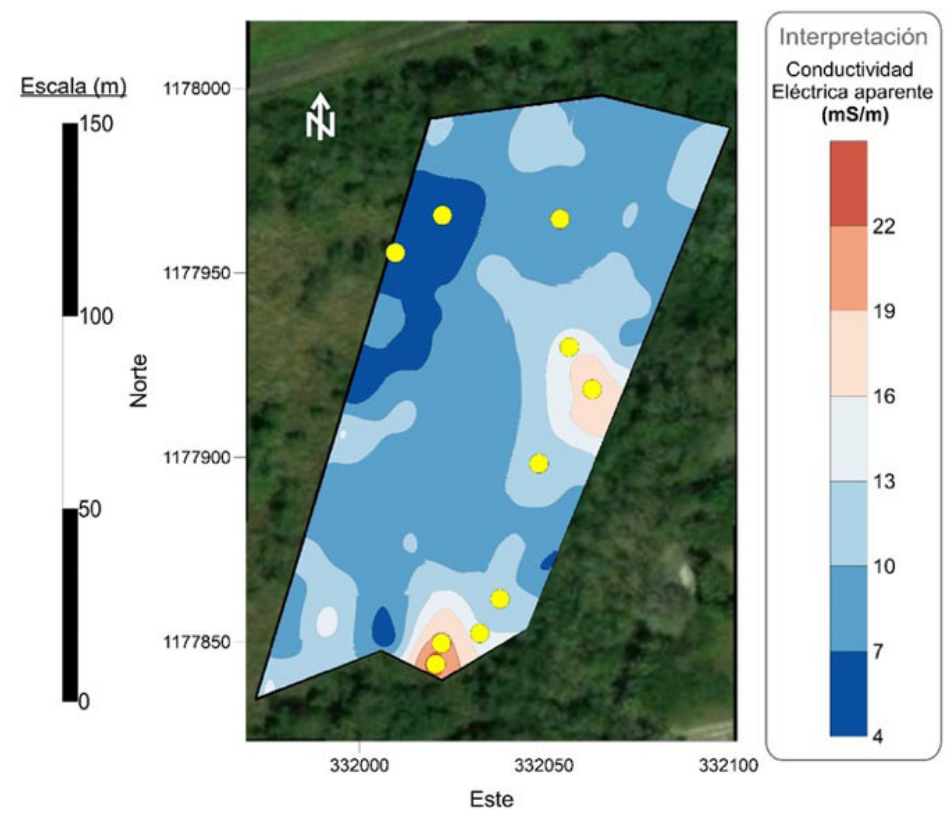

Figura 6. Mapa de conductividad eléctrica aparente superficial (CEa-sh, de 0 a $30 \mathrm{~cm}$ de profundidad) y puntos de muestreo en amarillo para calibración en el campus La Flor de la Universidad EARTH, denominado CAP (Centro de Agricultura de Precisión), Liberia, Guanacaste, Costa Rica. 2017.

Figure 6. Shallow apparent electrical conductivity map (ECa-sh, depth of 0 to $30 \mathrm{~cm}$ ) and calibration samples sites in yellow, located in campus La Flor of EARTH University, denomined CAP (Centro de Agricultura de Precisión), Liberia, Guanacaste, Costa Rica. 2017.

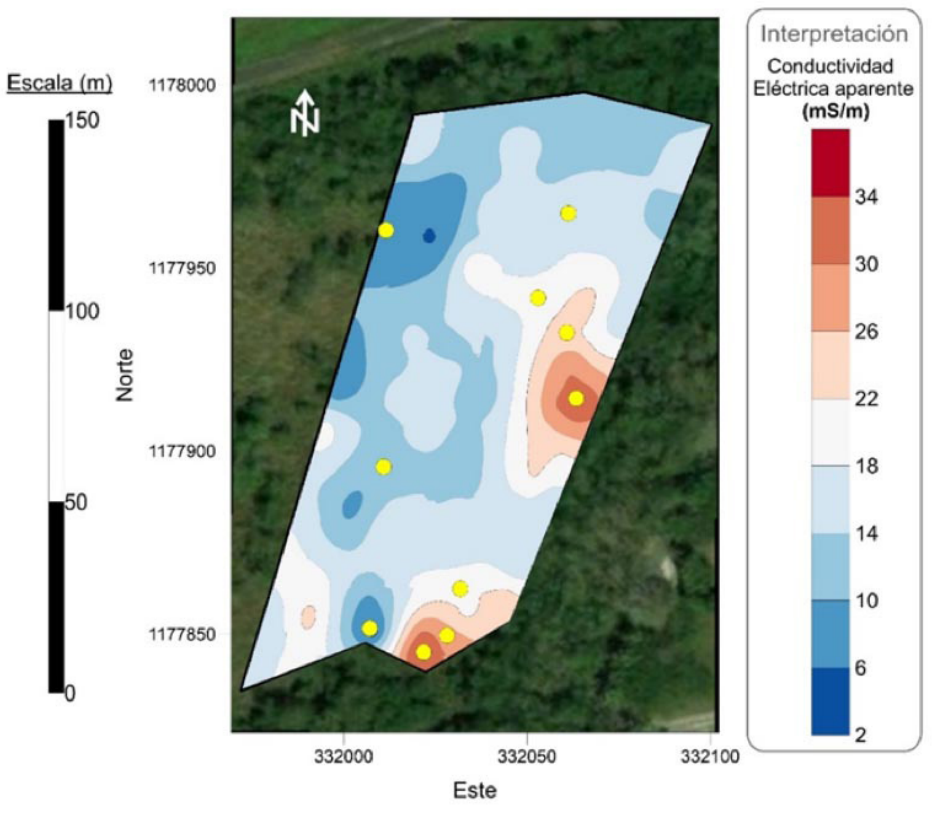

Figura 7. Mapa de conductividad eléctrica (CEa-dp, 30 a $90 \mathrm{~cm}$ de profundidad) y puntos de muestreo en amarillo para calibración en CAP (Centro de Agricultura de Precisión), Liberia, Guanacaste, Costa Rica. 2017.

Figure 7. Deep apparent electrical conductivity map (ECa-sh, depth of 0 to $30 \mathrm{~cm}$ ) and calibration samples sites in yellow in CAP (Centro de Agricultura de Precisión), Liberia, Guanacaste, Costa Rica. 2017. 


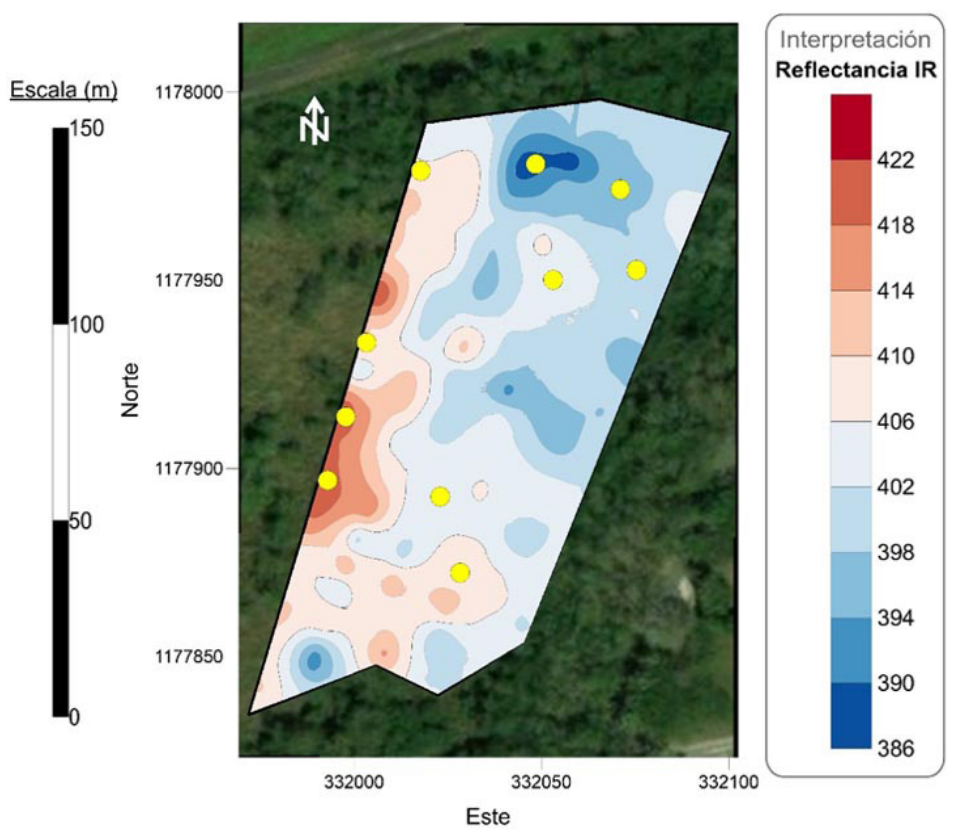

Figura 8. Mapa de reflectancia en infrarrojo cercano (NIR) y puntos de muestreo en amarillo para calibración en CAP (Centro de Agricultura de Precisión), Liberia, Guanacaste, Costa Rica. 2017.

Figure 8. Near-infrared reflectance (NIR) map and calibration samples sites in yellow in CAP (Centro de Agricultura de Precisión), Liberia, Guanacaste, Costa Rica. 2017.

Para el modo smc se recolectaron dieciocho muestras por hectárea en seis trayectos, con un distanciamiento entre trayectos de $15 \mathrm{~m}$ aproximadamente. Para su calibración se utilizaron diecisiete muestras. En el modo on the go se realizaron 61 lecturas por hectárea, en 6 trayectos y con una distancia aproximada de $15 \mathrm{~m}$ entre lecturas. Para la calibración se colectaron quince muestras.

Previo a la recolección de datos de pH con el equipo en los dos modos, se realizó la calibración de rutina con el software Veris Soil Viewer y buffers de pH 4 y 7 (Veris Technologies, 2017b). Se realizó el mapa de pH en modo on the go (Figura 9), al marcar los puntos de muestreo en contornos definidos para su respectiva calibración.

Para todos los puntos muestreados, se extrajeron los valores de los diferentes parámetros que mide el Veris MSP3 en los mapas interpolados, y con sus respectivos pares de laboratorio, se hicieron regresiones lineales simples y múltiples, lo que generó el coeficiente de determinación, siendo este el porcentaje de determinación en relación con la variable dependiente. La variable independiente (regresora) puede explicar la dependiente.

Debido a que, para MO, $\mathrm{N}$ y CIC, no hay una variable regresora dominante, se realizaron regresiones lineales múltiples, se utilizaron como regresoras: CEa-sh, CEa-dp, radio de CEa (CEa-sh/CEa-dp), NIR, vis-NIR, radio óptico (NIR/vis-NIR), curvatura, pendiente y elevación, con base en el mejor modelo con el programa digital InfoStat (Di-Renzo et al., 2017) a un nivel de significancia de 0,05. Las mismas regresoras se usaron para los componentes texturales, con el fin de obtener el mejor modelo.

Anterior a definir el modelo de regresión utilizado se realizó la eliminación de datos atípicos con el método de residuos estudentizados descrito por Montgomery et al. (2002), con el programa estadístico InfoStat (Di-Renzo et al., 2017). 


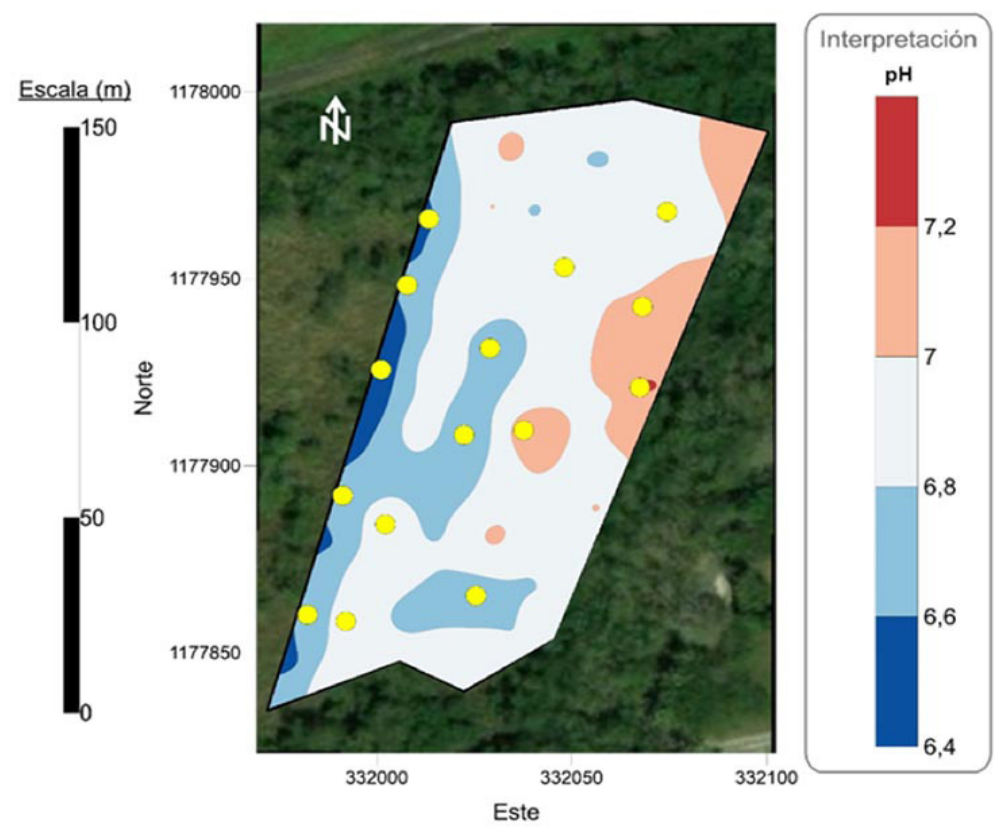

Figura 9. Mapa de pH modo on the go y puntos de calibración en amarillo en CAP (Centro de Agricultura de Precisión), Liberia, Guanacaste, Costa Rica. 2017.

Figure 9. $\mathrm{pH}$ map of on the go mode and samples sites calibration in yellow in CAP (Centro de Agricultura de Precisión), Liberia, Guanacaste, Costa Rica.

Otros coeficientes estadísticos que ayudaron a explicar la relación y precisión de la regresión lineal con sus pares de laboratorio fueron: i) el cuadrado de Pearson (R), que explica el grado de asociación entre dos variables, y ii) la raíz del error cuadrático medio de predicción (RECMP), que se define como en promedio el margen de error de las predicciones en las unidades del modelo.

Para CEa-sh y reflectancia en las dos localidades evaluadas, se realizó una validación cruzada de los modelos de predicción, esto consistió en: i) toma de muestras en grid (profundidad de 0 a 30), ii) análisis de laboratorio para cada parámetro (textura, materia orgánica, CIC total), iii) sobreponer los puntos de toma de muestras (grid) sobre los mapas interpolados generados con las lecturas del Veris MSP3, iv) extraer los datos de los mapas del Veris MSP3, v) calcular datos predichos utilizando la formula generada de la regresión lineal, v) calcular los coeficientes estadísticos: raíz del error cuadrático medio de validación cruzada (RECMVC) y el error medio absoluto (EMA) para pH. En el caso de CIC, N y MO, la validación cruzada se procedió por el método de validación cruzada dejando uno afuera.

\section{Resultados}

El método de calibración utilizado mostró resultados satisfactorios con la variable textura de suelo a una profundidad de 0 a $30 \mathrm{~cm}$ en ambas localidades (Cuadro 2). Logró coeficientes de determinación razonablemente precisos $\left(r^{2} \geq 0,55\right)$ (Sudduth et al., 2005) a excepción de la partícula de limo en el CAP. En el caso de la profundidad 
Cuadro 2. Estadística de la regresión lineal para conductividad eléctrica aparente superficial (CEa-sh) y profunda (CEa-dp), con partículas del suelo, en un estudio de calibración y validación del equipo Veris MSP3 en dos suelos de Guanacaste, Costa Rica. 2017. Table 2. Linear regression statistics for shallow apparent electrical conductivity (CEa-sh) and deep apparent electrical conductivity (CEA-dp) with soil particles, in a study of calibration and validation for Veris MSP3 equipment on two soils in Guanacaste, Costa Rica. 2017.

\begin{tabular}{|c|c|c|c|c|c|c|c|c|c|}
\hline \multirow{2}{*}{$\begin{array}{l}\text { Partícula } \\
\text { del suelo }\end{array}$} & \multirow[t]{2}{*}{ Ubicación } & \multicolumn{4}{|c|}{ CEa-sh } & \multicolumn{4}{|c|}{ CEa-dp } \\
\hline & & $\mathbf{n}$ & $\mathbf{R} \dagger$ & $\mathbf{R}^{2 \dagger}$ & RECMP $\dagger$ & $\mathbf{n}$ & $\mathbf{R} \dagger$ & $\mathbf{R}^{2} \dagger$ & RECMP†** \\
\hline$\%$ Arcilla & Costeña & 12 & 0,75 & 0,56 & 7,03 & 13 & 0,75 & 0,56 & 16,09 \\
\hline$\%$ Arena & Costeña & 12 & $-0,65$ & 0,42 & 6,63 & 13 & $-0,69$ & 0,48 & 19,15 \\
\hline \% Limo & Costeña & 12 & $-0,86$ & 0,74 & 1,45 & 13 & $\mathrm{~N} / \mathrm{S}$ & $\mathrm{N} / \mathrm{S}$ & $\mathrm{N} / \mathrm{S}$ \\
\hline$\%$ Arcilla & CAP & 9 & 0,80 & 0,64 & 6,54 & 9 & $0,59 \neq$ & $0,35 \neq$ & $8,46 \neq$ \\
\hline$\%$ Arena & CAP & 9 & $-0,91$ & 0,82 & 2,29 & 9 & $\mathrm{~N} / \mathrm{S}$ & $\mathrm{N} / \mathrm{S}$ & $\mathrm{N} / \mathrm{S}$ \\
\hline \% Limo & CAP & 9 & $\mathrm{~N} / \mathrm{S}$ & $\mathrm{N} / \mathrm{S}$ & $\mathrm{N} / \mathrm{S}$ & 9 & 0,67 & 0,45 & 6,30 \\
\hline
\end{tabular}

$\dagger$ valores significativos a $\mathrm{p}<0,05$, $\neq$ valores significativos a $\mathrm{p}<0,1 \mathrm{~N} / \mathrm{S}$ valores no significativos a $\mathrm{p}<0,1 / \uparrow$ significant values at $\mathrm{p}<0.05$, $\neq$ significant values at $\mathrm{p}<0.1, \mathrm{~N} / \mathrm{S}$ not significant values at $\mathrm{p}<0.1$.

*Raíz del error cuadrático medio de predicción / Root mean square error.

30 a $90 \mathrm{~cm}$, a excepción de la arcilla en Costeña, los coeficientes de determinación no fueron suficientemente precisos $\left(\mathrm{r}^{2} \leq 0,55\right)$.

Las mejores regresiones (coeficientes de determinación más altos) entre las partículas de suelo y conductividad eléctrica aparente (Figura 10) por profundidad y localidad, fueron para arena en CAP a una profundidad de 0 a 30 cm y arcilla en Costeña a una profundidad de 30 a $90 \mathrm{~cm}$.

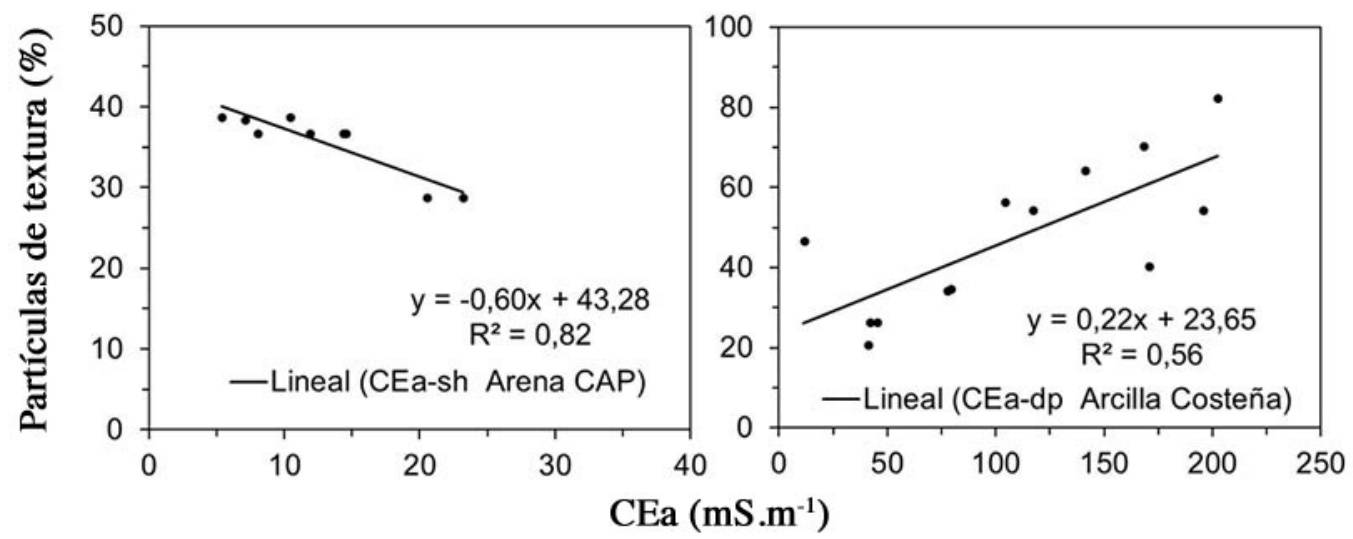

Figura 10. Mejores regresiones entre conductividad eléctrica aparente (CEa) y tamaño de partículas de suelo en dos profundidades, en un estudio de calibración y validación del equipo Veris MSP3 en dos suelos de Guanacaste, Costa Rica. 2017.

Figure 10. The best regressions between apparent electrical conductivity (CEa) and soil particle size in two depths, in a study of calibration and validation of Veris MSP3 equipment on two soils in Guanacaste, Costa Rica. 2017. 
La validación cruzada de las ecuaciones de predicción de las partículas de suelo en profundidad 0 a $30 \mathrm{~cm}$ en las dos localidades mostraron un margen de error razonable (similar al RECMP), por lo que, los datos predichos de las partículas de suelo a partir de la CEa-sh fueron cercanos al dato real (mayor error promedio fue de 6,92) (Cuadro 3).

Cuadro 3. Validación cruzada de partículas de suelo a partir de conductividad eléctrica aparente superficial (CEa-sh) en los terrenos Costeña y CAP (Centro de Agricultura de Precisión), en un estudio de calibración y validación del equipo Veris MSP3 en dos suelos de Guanacaste, Costa Rica. 2017.

Table 3. Cross-validation of soil particles from shallow aparent electrical conductivity (CEa-sh) in Costeña and CAP (Centro de Agricultura de Precisión) fields, in a study of calibration and validation of Veris MSP3 equipment on two soils in Guanacaste, Costa Rica. 2017.

\begin{tabular}{cccc}
\hline & & \multicolumn{2}{c}{ CEa-sh } \\
\cline { 3 - 4 } Propiedad del suelo & Ubicación & n & RECMVC* \\
\hline \% Arcilla & Costeña & 15 & 6,92 \\
\% Arena & Costeña & 15 & 6,85 \\
\% Limo & Costeña & 15 & 3,31 \\
\% Arcilla & CAP & 10 & 5,25 \\
\% Arena & CAP & 10 & 5,10 \\
Limo & CAP & 10 & 4,60 \\
\hline
\end{tabular}

* Raíz del error cuadrático medio de validación cruzada / Root mean square error of cross validation.

Con respecto a los parámetros CIC total, y $\mathrm{N}(\%)$ no se establecieron coeficientes de determinación significativos $\left(\mathrm{r}^{2} \geq 0,55\right)$, no siendo el caso de la materia orgánica con 0,61 , con el empleo de una regresión lineal múltiple (radio óptico y pendiente como regresoras) (Cuadro 4) (Figura 11).

Cuadro 4. Coeficientes estadísticos para regresiones lineales de capacidad de intercambio catiónico total (CIC total), materia orgánica (MO) y N, en el estudio de calibración y validación del equipo Veris MSP3 en dos de Guanacaste, Costa Rica. 2017.

Table 4. Statistical coefficients for the linear regressions of total cation exchange capacity (total CIC), organic matter (OM), and $\mathrm{N}$ in the study of calibration and validation of Veris MSP3 equipment on two soils in Guanacaste, Costa Rica. 2017.

\begin{tabular}{|c|c|c|c|c|c|c|c|}
\hline \multirow{2}{*}{$\begin{array}{c}\text { Propiedad } \\
\text { del suelo }\end{array}$} & \multirow[b]{2}{*}{ Localidad } & \multirow[b]{2}{*}{ Regresión } & \multirow[b]{2}{*}{ Regresoras } & \multirow[b]{2}{*}{$\mathbf{n}$} & \multirow[b]{2}{*}{$\mathbf{R} \dagger$} & \multirow[b]{2}{*}{$\mathbf{R}^{2 \dagger}$} & \multirow[b]{2}{*}{ RECMP†* } \\
\hline & & & & & & & \\
\hline CIC Total & Costeña & Simple & CEa-sh & 22 & 0,69 & 0,48 & 4,96 \\
\hline $\mathrm{N}(\%)$ & CAP & Simple & CEa-dp & 19 & $-0,57$ & 0,32 & 0,49 \\
\hline $\mathrm{MO}(\%)$ & CAP & Múltiple & $\begin{array}{l}\text { Radio Óptico } \\
\text { Pendiente }\end{array}$ & 19 & $\begin{array}{r}0,60 \\
-0,54\end{array}$ & 0,61 & 1,57 \\
\hline
\end{tabular}

$\dagger$ valores significativos a $\mathrm{p}<0,05 / \uparrow$ significant values at $\mathrm{p}<0.05$.

*Raíz del error cuadrático medio de predicción / Root mean square error.

La validación cruzada de MO confirmó un buen modelo de predicción, al obtener un RECMVC de 1,76 (similar al RECMP obtenido en la ecuación de predicción) (Cuadro 5).

El pH smc presentó correlaciones bajas con $\mathrm{pH}$ en agua y significativas $\left(\mathrm{r}^{2} \geq 0,55\right)$ con $\mathrm{pH}$ en $\mathrm{KCl}$ (Figura 12), ya el $\mathrm{pH}$ modo on the go solo presentó correlaciones significativas $\left(\mathrm{r}^{2} \geq 0,55\right)$ con $\mathrm{pH}$ en $\mathrm{KCl}$. La mejor correlación 


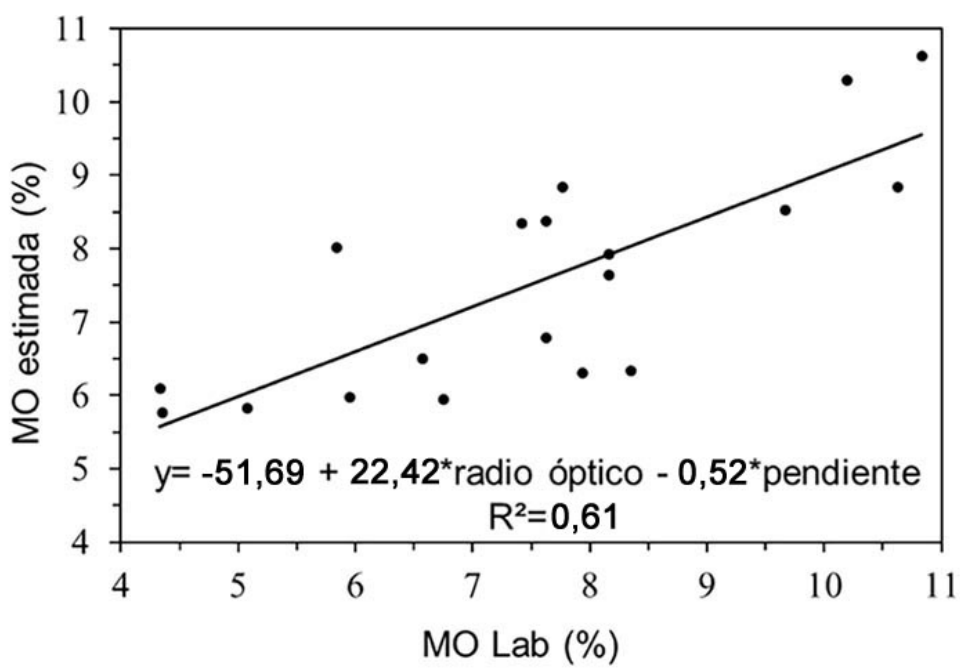

Figura 11. Relación entre materia orgánica (MO) de laboratorio y MO estimada por regresión lineal múltiple en CAP (Centro de Agricultura de Precisión), en el estudio de calibración y validación del equipo Veris MSP3 en dos suelos de Guanacaste, Costa Rica. 2017.

Figure 11. Relation between laboratory organic matter $(\mathrm{OM})$ and $\mathrm{OM}$ estimate by the multiple linear regression in CAP (Centro de Agricultura de Precisión), in a study of calibration and validation of Veris MSP3 equiment on two soils in Guanacaste, Costa Rica. 2017.

Cuadro 5. Validación cruzada de capacidad de intercambio catiónico (CIC) y materia orgánica (MO) por el método de leave one out cross validation en CAP (Centro de Agricultura de Precisión), en un estudio de calibración y validación del equipo Veris MSP3 en dos suelos de Guanacaste, Costa Rica, 2017.

Table 5. Cross-validation of the cation exchange capacity (CIC) and organic matter (MO) by the leave one out cross validation method in CAP (Centro de Agricultura de Precisión), in a study of calibration and validation of Veris MSP3 equipment on two soils in Guanacaste, Costa Rica. 2017.

\begin{tabular}{ccccccc}
\hline $\begin{array}{c}\text { Propiedad del } \\
\text { Suelo }\end{array}$ & Ubicación & Regresión lineal & Regresoras & $\begin{array}{c}\text { Rango de datos } \\
\text { de regresoras }\end{array}$ & n & RECMVC* \\
\hline MO $(\%)$ & CAP & Múltiple & $\begin{array}{c}\text { Radio óptico y } \\
\text { pendiente }\end{array}$ & $\begin{array}{c}2,60 \text { a } 2,82 \\
0,69 \text { a } 7,04\end{array}$ & 19 & 1,76 \\
\hline
\end{tabular}

$\dagger$ valores significativos a $\mathrm{p}<0,05 / \uparrow$ significant values at $\mathrm{p}<0.05$.

* Raíz del error cuadrático medio de validación cruzada / Root mean square error of cross validation.

fue con el modelo de $\mathrm{pH}$ en $\mathrm{KCl}$ en smc con una asociación positiva de 0,77 , un $\mathrm{r}^{2}$ de 0,59 y un RECMP de 0,07 (Cuadro 6).

Para la validación cruzada (Cuadro 7), la precisión de los modelos de $\mathrm{pH}$ semi controlado y on the go fue la misma $(0,09)$, en cambio la precisión del modelo semi controlado para pH en agua fue muy baja con 0,25 (Cuadro 6). 


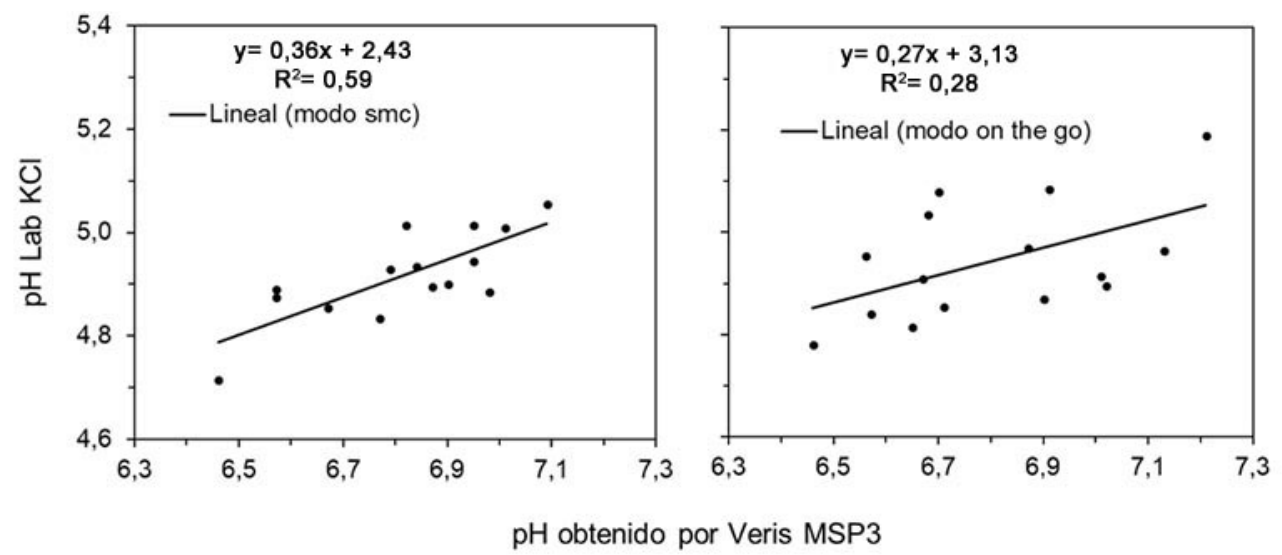

Figura 12. pH en $\mathrm{KCl}$ modo semicontrolado (smc) y on the go en el terreno del Centro de Agricultura de Precisión (CAP) de la Universidad EARTH, en un estudio de calibración y validación del equipo Veris MSP3 en dos suelos de Guanacaste, Costa Rica. 2017.

Figure 12. $\mathrm{KCl} \mathrm{pH}$ on semi-controlled $(\mathrm{smc})$ and on the go mode in $\mathrm{CAP}$ (Centro de Agricultura de Precisión) of EARTH University in the study of calibration and validation of Veris MSP3 equiment on two soils in Guanacaste, Costa Rica. 2017.

Cuadro 6. Estadística de la regresión lineal para pH obtenido por Veris MSP3 y pH laboratorio, en un estudio de calibración y validación del equipo Veris MSP3 en dos suelos de Guanacaste, Costa Rica, 2017.

Table 6. Linear regressions statistical for $\mathrm{pH}$ obtained by Veris MSP3 and lab $\mathrm{pH}$ in a study of calibration and validation of Veris MSP3 equipment on two soils in Guanacaste, Costa Rica. 2017.

\begin{tabular}{ccccccc}
\hline Propiedad del suelo & Modo & \multicolumn{4}{c}{ pH obtenido por Veris MSP3 } \\
\cline { 3 - 7 } & & $\mathbf{n}$ & Rango de datos Veris & $\mathbf{R} \dagger$ & $\mathbf{R}^{2} \dagger$ & RECMP $\dagger *$ \\
\hline pH en agua & Semi-controlado & 17 & 6,46 a 7,13 & 0,73 & 0,53 & 0,1 \\
pH en Cloruro de Potasio & Semi-controlado & 15 & 6,46 a 7,13 & 0,77 & 0,59 & 0,07 \\
pH en Cloruro de Potasio & on the go & 15 & 6,46 a 7,21 & 0,53 & 0,28 & 0,1 \\
\hline
\end{tabular}

$\dagger$ valores significativos a $\mathrm{p}<0,05 / \uparrow$ significant values at $\mathrm{p}<0.05$.

*Raíz del error cuadrático medio de predicción / Root mean square error.

Cuadro 7. Validación cruzada para pH obtenidos por el Veris MSP3, en el estudio de calibración y validación del equipo Veris MSP3 en dos suelos de Guanacaste, Costa Rica, 2017.

Table 7. Cross-validation of $\mathrm{pH}$ obtained by Veris MSP3, in the study of calibration and validation of Veris MSP3 equiment on two soils in Guanacaste, Costa Rica.

\begin{tabular}{ccccc}
\hline Propiedad del suelo & Modo & \multicolumn{3}{c}{ pH Veris } \\
\cline { 3 - 5 } & & $\mathbf{n}$ & EMA & RECMVC* \\
\hline pH en agua & Semi-controlado & 10 & 0,23 & 0,25 \\
pH en Cloruro de Potasio & Semi-controlado & 10 & 0,06 & 0,09 \\
pH en Cloruro de Potasio & on the go & 10 & 0,06 & 0,09 \\
\hline
\end{tabular}

*Raíz del error cuadrático medio de validación cruzada / Root mean square error of cross validation. 


\section{Discusión}

Existe una fuerte correlación entre CEa y las partículas del suelo, cuando se trata de suelos no salinos (caso del presente estudio) (Williams y Hoey, 1987). En CEa-sh al trabajar con Veris 3100, Sudduth et al. (2003) encontraron un $\mathrm{r}^{2}$ de 0,62 a 0,77 para arcilla y 0,60 a 0,79 para limo, asimismo, Sudduth et al. (2005) obtuvieron un $\mathrm{r}^{2}$ para arcilla de 0,37 a 0,63 y 0,39 para limo. Los resultados encontrados en dichas investigaciones se encuentran en el mismo rango del presente estudio. En ambos casos no se reportó correlación con arena. En investigaciones efectuadas por Sudduth et al. $\left(2003\right.$; 2005) se obtuvieron $\mathrm{r}^{2}$ similares al del presente estudio para arcilla y limo con CEa-dp.

En un trabajo de regresión lineal múltiple, en que se combinaron datos de CEa del equipo EM38MK2, reflectancia del espectro visible e infrarrojo cercano, fuerza de inserción, CEa del Veris 4000, además de rayos gamma naturales del equipo The Mole, para predecir los componentes de textura y materia orgánica, Wetterlind et al. (2015) concluyeron que al combinar los sensores hubo una leve mejora en todas las correlaciones, a excepción de la arcilla en una de las parcelas evaluadas. Los mismos autores encontraron que los RECMP de textura fueron de 5,6 para arcilla, 6,1 para limo y 9,1 para arena. Dichos resultados fueron similares a los obtenidos en el presente experimento, a excepción de arcilla y arena con CEa-dp en Costeña, que resultaron con baja precisión. Además, a diferencia de Wetterlind et al. (2015), en la presente investigación los mejores modelos fueron de regresiones lineales simples. Otros autores realizaron regresiones lineales simples para predecir textura a través de CEa por inducción utilizando el equipo EM38, y obtuvieron $\mathrm{r}^{2}$ aceptables y semejantes al presente estudio (Lesch et al., 2005; Weller et al., 2007; Heil y Schmidhalter, 2010; Rodríguez et al., 2011).

Varios autores encontraron un $\mathrm{r}^{2}$ para CIC mayor al del presente estudio, entre estos Sudduth et al. (2005), presentaron coeficientes de determinación de 0,71 al analizar seis fincas, mientras que Kweon et al. (2013), en dos fincas diferentes, obtuvieron un coeficiente de determinación de 0,40 y 0,96 . Otras investigaciones como Machado et al. (2015) correlacionaron CIC con CEa por inducción, obteniendo coeficientes de determinación con regresiones lineales simples de $0,56,0,67$ y 0,78 en sus respectivas frecuencias de $2 \mathrm{~Hz}, 7 \mathrm{~Hz}$ y $15 \mathrm{~Hz}$ con el equipo GSSI Profiler EMP-400 en un suelo Oxisol. La precisión encontrada en dicho estudio fue mayor al obtenido en la presente investigación, con un RECMP de 4,96. Por su parte Kweon et al. (2013) obtuvieron RECMP de 1,57 y 0,64.

Cho et al. (2017), al analizar diversos componentes del suelo con el equipo Veris P4000, a través espectroscopía de reflectancia difusa con vis-NIR y NIR con el método de parcial mínimo cuadrado (PLS, en inglés), encontraron para $\mathrm{N}$ un coeficiente de determinación de 0,77 y una precisión de validación de 0,02 . Además, al analizar esta longitud de banda con el equipo AgroSpec y aplicar los modelos de componente principal de regresión (PCR, en inglés) y regresión de parciales mínimos cuadrados (PLSR, en inglés), Morellos et al. (2016) encontraron $\mathrm{r}^{2}$ de 0,73 y 0,78 , RECMP de 0,0076 y 0,0786 , además de una RECMCV de 0,0061 y 0,0068 para PCR y PLSR, respectivamente. Debido a que la correlación de CEa-dp con nitrógeno fue muy baja, no se realizó validación cruzada para este parámetro en el presente estudio.

El valor del coeficiente para MO estuvo dentro del rango encontrado por otros autores como Kweon et al. (2013), que al analizar la MO con diferentes tipos de regresoras presentaron coeficientes de determinación que variaron de 0,37 a 0,99. Knadel et al. (2015) analizando la correlación de carbono orgánico del suelo, con reflectancia a partir de regresión lineal simple y múltiple con vis-NIR y NIR encontraron $\mathrm{r}^{2}$ de 0,35 a 0,94 . En cambio, Machado et al. (2015) para materia orgánica estimada a partir de CEa inducida, encontraron coeficiente de determinación de 0,41, 0,24 y 0,30 para las frecuencias de $2 \mathrm{~Hz}, 7 \mathrm{~Hz}$ y $15 \mathrm{~Hz}$, respectivamente, siendo estos coeficientes menores al del presente estudio.

En un estudio con MO, Kweon et al. (2013) tuvieron una mayor precisión predicha que varió entre 0,04 a 0,91, y de validación cruzada de 0,07 a 1,83, igualmente, Knadel et al. (2015) presentaron una precisión del modelo por validación cruzada de 0,14 a 0,69 , siendo estas más precisas que la presentada en esta investigación con raíz del error cuadrático medio de validación cruzada (RECMVC) de 1,76. 
Sethuramasamyraja et al. (2008) utilizando el ion selectivo para $\mathrm{pH}$, potasio soluble y nitrato residual en modo on the go, mostró un rango de $\mathrm{r}^{2}$ de 0,85 a 0,89 con sus respectivas muestras de $\mathrm{pH}$ laboratorio, mientras que Adamchuk et al. (2007) con el equipo Veris en modo on the go en Estados Unidos presentaron correlaciones con $\mathrm{r}^{2}>0,5$.

Mejores resultados se han reportado, pero con diferentes métodos; por ejemplo, Schirrmann et al. (2011) en suelos alcalinos de Alemania hallaron un coeficiente de determinación de 0,92 entre $\mathrm{pH}$ extraído con $\mathrm{CaCl}_{2}$ y en modo semi-controlado.

Medias de errores absolutos (EMA) de 0,32 a 1,09 las obtuvieron Schirrmann et al. (2011), mientras que Adamchuk et al. (2007) presentaron medias de errores absolutos de 0,07 a 0,44. La EMA del presente estudio fue más baja que los reportes de las investigaciones anteriores y variaron entre 0,06 y 0,23 .

\section{Conclusiones}

El método de calibración utilizado obtuvo correlaciones razonablemente precisas $\left(R^{2} \geq 0,55\right)$ para las variables textura de suelo en la profundidad de 0 a $30 \mathrm{~cm}$ y materia orgánica. Sin embargo, para textura de suelo de 30 a 90 $\mathrm{cm}, \mathrm{N}$, CIC total y $\mathrm{pH}$; se debe considerar otra metodología de calibración, debido a que obtuvieron correlaciones no precisas $\left(\mathrm{R}^{2} \leq 0,55\right)$.

\section{Literatura citada}

Adamchuk, V.I., E.D. Lund, T.M. Reed, and R.B. Ferguson. 2007. Evaluation of an on-the-go technology for soil pH mapping. Precision Agric. 8(3):139-149. doi:10.1007/s11119-007-9034-0

Adamchuk, V.I., M. Morgan, and D.R Ess. 1999. An automated sampling system for measuring soil pH. Trans. ASABE 42:885892. doi: $10.13031 / 2013.13268$

Bianchini, A.A., and Mallarino A.P. 2002. Soil sampling alternatives and variable-rate liming for a soybean-corn rotation. Agro J. 94(6):1355-1366 doi:10.2134/agronj2002.1355

Cho, Y., A.H. Sheridan, K.A. Sudduth, and K.S. Veum. 2017. Comparison of field and laboratory VNIR spectroscopy for profile soil property estimation. Trans. ASABE. 60:1503-1510. doi:10.13031/trans.12299

CIA (Centro de Investigaciones Agronómicas). 2016. Mapa digital de suelos de Costa Rica CIA, San José, CRI. http://www.cia. ucr.ac.cr/?page_id=139 (consultado 6 feb. 2017).

Corwin, D.L. 2005. Geospatial measurement of apparent soil electrical conductivity for characterizing soil spatial variability. In: J. Álvarez, and R. Munoz, editors, Soil-water-solute process characterization: an integrated approach. CRC Press, FL, USA. p. 639-664.

Corwin, D.L., and S.M. Lesch. 2005. Characterizing soil spatial variability with apparent soil electrical conductivity. Part I. Survey protocols. Comput. Electron. Agric. 46:135-152. doi:10.1016/j.compag.2004.11.002

Di-Rienzo, J.A., F. Casanoves, M.G. Balzarini, L. González, M. Tablada, y C.W. Robledo. 2017. InfoStat: software estadístico 2017. Universidad Nacional de Córdoba, ARG.

Google Earth. 2017. Versión 7.1.8.3036. Google, Mountain View, CA, USA.

Grisso, R.B., M. Alley, D. Holshouser, and W. Thomason. 2009. Precision farming tools: Soil electrical conductivity. DOCPLAYER. https://docplayer.net/10831185-Precision-farming-tools-soil-electrical-conductivity.html (accessed May 6, 2017). 
Heil, K., and U. Schmidhalter. 2010. Characterization of soil texture variability using the apparent soil electrical conductivity at a highly variable site. Comput. Geosci. 39:98-110. doi: 10.1016/j.cageo.2011.06.017

Hernández, C.A., W.A. Barrera, E. Spaans, y R. Vaquero. 2006. Agricultura de precisión en la empresa agrocomercial EARTH: fertilización dinámica. Tierra Trop. 2(2):177-185.

IMN (Instituto Meteorológico Nacional). 2017. Datos climáticos. IMN, CRI. https://www.imn.ac.cr/web/imn/inicio (consultado 7 feb. 2017).

Johnson, C.K., J.W. Doran, H.R. Duke, B.L. Weinhold., K.M. Eskridge, and J.F. Shanahan. 2001. Field-scale electrical conductivity mapping for delineating soil condition. Soil Sci. Am. J. 65:1829-1837.

Knadel, M., A. Thomsen, K. Schelde, and M.H. Greve. 2015. Soil organic carbon and particle sizes mapping using vis-NIR, EC and temperature mobile sensor platform. Comp. Electron. Agric. 114:134-144. doi:10.1016/j.compag.2015.03.013

Kweon, G., E. Lund, and C. Maxton. 2013. Soil organic matter and cation-exchange capacity sensing with on-the-go electrical conductivity and optical sensors. Geoderma 199:80-89. doi:10.1016/j.geoderma.2012.11.001

Lesch, S.M., D.L. Corwin, and D.A Robinson. 2005. Apparent soil electrical conductivity mapping as an agricultural management tool in arid zone soils. Comp. Electron. Agric. 46:351-378. doi: 10.1016/j.compag.2004.11.007

Machado, F.C., R. Montanari, L.S Shozo, L.H. Lovera, and E.D Lima. 2015. Spatial dependence of electrical conductivity and chemical properties of the soil by electromagnetic induction. Rev. Bras. Ciênc. Solo 39:1112-1120. doi:10.1590/010006 83rbcs 20140794

Maxton, C., P. Drummond, E. Lund, and K. Jensen. 2017. Mobile soil optical mapping system. Patent 9,743,574. Veris Technologies Inc., USA.

Melo, J.A., J.L. Loratte, E.R. Alves, R. Negrão, and J.L. Morelli. 2014. Precision agriculture for sugarcane management: a strategy applied for brazilian conditions. Acta Sci. Agron. 36:111-117. doi:10.4025/actasciagron. v36i1.17664.

Montgomery, D.C., E.A. Peck, y G.G. Vining. 2002. Introducción al análisis de regresión lineal. $3^{\text {ra }}$ ed. Compañía Editorial Continental, México, DF. MEX.

Morellos, A., X.E. Pantazi, D. Moshou, T. Alexandridis, R. Whetton, G. Tziotzios, and A.M. Mouazen. 2016. Machine learning based prediction of soil total nitrogen, organic carbon and moisture content by using VIS-NIR spectroscopy. Biosyst. Eng. 152:104-116. doi:10.1016/j.biosystemseng.2016.04.018

Mulla, D.J., and McBratney A.B 2000. Soil spatial variability. In: M.E. Summer, editor, Handbook of soil science. CRC Press, FL, USA, p. A321-A352.

Rodriguez, JR., R.E. Plant, J.J Lambert, and D.R. Smart. 2011. Using apparent soil electrical conductivity (ECa) to characterize vineyard soils of high clay content. Precision Agric. 12:775-794. doi:10.1007/s11119-011-9220-y

Schirrmann, M., R. Gebbers, E. Kramer, and J. Seidel. 2011. Soil pH mapping with an on-the-go sensor. Sensors (Basel) 11:573598. doi:10.3390/s 110100573

Sethuramasamyraja, B., V.I. Adamchuk, A. Dobermann, D.B Marx, D.D. Jones, and G.E. Meyer. 2008. Agitated soil measurement method for integrated on-the-go mapping of soil $\mathrm{pH}$, potassium and nitrate contents. Comp. Electron. Agric. 60:212-225. doi:10.1016/j.compag.2007.08.003

Sudduth, K.A., N.R. Kitchen, G.A. Bollero, D.G. Bullock, and W.J. Wiebold. 2003. Comparison of electromagnetic induction and direct sensing of soil electrical conductivity. Agron. J. 95:472-482. doi:10.2134/agronj2003.4720 
Sudduth, KA., N.R. Kitchen, W.J. Wiebold, W.D. Batchelor, G.A. Bollero, D.G. Bullock, and K.D. Thelen. 2005. Relating apparent electrical conductivity to soil properties across the north-central USA. Comput. Electron. Agric. 46:263-283. doi:10.1016/j.compag.2004.11.010

Surfer. 2017. Golden software. 13. Golden Surfer, Golden, CO, USA.

Veris Technologies. 2017a. Veris MSP3 EC/pH/OM Parts Manual. Veris Technologies, Salina, KS, USA. https://www.veristech. com/more/support/manuals (consultado 6 nov 2017).

Veris Technologies. 2017b. Veris Soil Viewer 2.87. Veris Technologies, Salina, KS, USA.

Weller, U., M. Zipprich, M. Sommer, W.Z. Castell, and M. Wehrhan. 2007. Mapping clay content across boundaries at the landscape scale with electromagnetic induction. Soil Sci. Soc. Am. J. 71:1740-1747. doi:10.2136/sssaj2006.0177

Wetterlind, J., K. Piikki, B. Stenberg, and M. Söderström. 2015. Exploring the predictability of soil texture and organic matter content with a commercial integrated soil profiling tool. Eur. J. Soil Sci. 66:631-638. doi:10.1111/ejss.12228

Williams, B.G., and D. Hoey. 1987. The use of electromagnetic induction to detect the spatial variability of the salt and clay contents of soils. Soil Res. 25:21-27. doi:10.1071/SR9870021

Yao, R., J. Yang, D. Wu, W. Xie, P. Gao, and W. Jin. 2016. Digital mapping of soil salinity and crop yield across a coastal agricultural landscape using repeated electromagnetic induction (EMI) surveys. PloS One 11(5):e0153377. doi:10.1371/ journal.pone. 0153377 